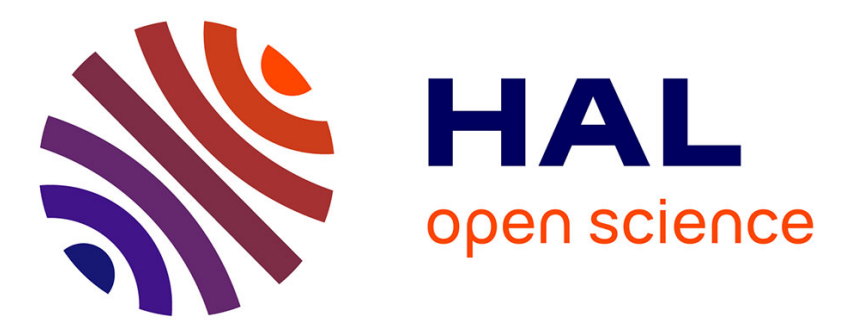

\title{
Application of a continuous supervisory fuzzy control on a discrete scheduling of manufacturing systems.
}

Karim Tamani, Reda Boukezzoula, Georges Habchi

\section{To cite this version:}

Karim Tamani, Reda Boukezzoula, Georges Habchi. Application of a continuous supervisory fuzzy control on a discrete scheduling of manufacturing systems.. 8ème Conf. Internationale de MOdélisation et SIMulation (MOSIM'10), May 2010, Hammamet, Tunisia. pp.CD. hal-00586426

\section{HAL Id: hal-00586426 \\ https://hal.univ-smb.fr/hal-00586426}

Submitted on 28 Sep 2011

HAL is a multi-disciplinary open access archive for the deposit and dissemination of scientific research documents, whether they are published or not. The documents may come from teaching and research institutions in France or abroad, or from public or private research centers.
L'archive ouverte pluridisciplinaire HAL, est destinée au dépôt et à la diffusion de documents scientifiques de niveau recherche, publiés ou non, émanant des établissements d'enseignement et de recherche français ou étrangers, des laboratoires publics ou privés. 


\title{
APPLICATION OF A CONTINUOUS SUPERVISORY FUZZY CONTROL ON A DISCRETE SCHEDULING OF MANUFACTURING SYSTEMS
}

\section{K. TAMANI, R. BOUKEZZOULA}

LISTIC / Polytech'Savoie

Domaine Universitaire - BP 80439

74944 Annecy le Vieux Cedex - France

\{karim.tamani, reda.boukezzoula\}@univ-savoie.fr

\section{G. HABCHI}

SYMME / Polytech'Savoie

Domaine Universitaire - BP 80439

74944 Annecy le Vieux Cedex - France

georges.habchi@univ-savoie.fr

\begin{abstract}
This paper considers the modelling and simulation of a hierarchical production-flow control system. Particularly, the system capacity allocation has been addressed by a set of distributed and supervised fuzzy controllers. The objective is to adjust the machine's production rates in such a way that satisfies the demand while maintaining the overall performances within acceptable limits. Given the adjusted production rates, the problem of scheduling of jobs is considered at the shop-floor level. In this case, the actual dispatching times are determined from the continuous production rates through a sampling procedure. To deal with conflicts between jobs at a shared machine, a decision for the actual part to be processed is taken using some criteria which represent a measure of the job's priority. A case study demonstrates the efficiency of the proposed control approach
\end{abstract}

KEYWORDS: Manufacturing Systems, Distributed Fuzzy Control, Supervisory Control, Fuzzy intervals, Scheduling.

\section{INTRODUCTION}

Production-flow control of manufacturing systems involves decision making such as part release, routing, production orders scheduling and set-up times, etc. with the objective of producing the customer demands in needed times and with minimum costs. Particularly, in scheduling problems, the objective is to find a way to assign and sequence the use of shared resources such that production constraints are satisfied. However, the complexity and the importance of scheduling problems have concentrated the efforts of different research communities, concerned with artificial intelligence (Grabot, 2001), dynamic programming, system simulation (Habchi and Berchet, 2003) and control theory (Cho and Prabhu, 2000; Gershwin, 2000; Wiendahl and Breithaupt, 2000).

More precisely, the scheduling of job-shop manufacturing systems with flexible machines and producing multiple part types has been studied by many approaches. The most developed ones have been enumerative algorithms that provide exact solutions either by means of elaborate and sophisticated mathematical constructs, such as linear and constraint programming (Pinedo, 2002; Sanghoon and Mooyoung, 2003), or by means of the branch and bound enumerative strategy, which involves search of a dynamically constructed tree that represents the solution space (Brucker et al., 1994). However, the limitations of the enumerative techniques have led to suboptimal approximation methods using simulation with priority dispatching rules (Chan et al., 2003). Furthermore, in the case of incomplete or imprecise data knowledge such as task durations and due dates, some solutions for scheduling problems have been provided according to artificial intelligence techniques, including neural networks, fuzzy logic, evolutionary algorithm and agent-based systems (Akyol and Bayhan, 2007; Dang and Frankovic, 2002; Dubois et al., 2003). The research reported in this paper is based on this last idea where a fuzzy logic is used in a distributed and supervised control strategy for discrete scheduling problems. Indeed, given a job-shop manufacturing system, this research attempts to address, at the shop-floor level, the discrete dispatching of the machine production rates (production capacity) allocated at the flow control level. In this case, the proposed approach uses continuous control theory and artificial intelligence techniques for production flow regulation of realistic (in terms of modelling assumptions) manufacturing systems (Rovithakis et al., 1999; Tamani et al., 2009; Tsourveloudis et al., 2007).

In our previous work, a supervised control strategy with a set of distributed fuzzy controllers has been developed for production-flow regulation. The originality of the proposed methodology resides in the supervisory based fuzzy arithmetic interval which significantly improves the overall system performances. Indeed, the supervisor combines multiple and possibly conflicting objectives such that a best compromise can be achieved between them. In this case, the overall objectives are quantified by fuzzy intervals since they are specified as imprecise and uncertain information. The provided supervisory control actions aim to compensate the myopic of the distributed local control when the overall system performances deviate from their objectives.

When a flow control methodology is assumed, the objective is to perform a scheduling problem in order to manage the transient from the flow control (continuous) to the shop-floor level (discrete). For this purpose, the 
developed scheduler is based on sampling procedure which translates the continuous-time production rates, computed at the flow control level, to a series of loading times at the shop-floor level. Moreover, in the context of multiple product systems, the selection of the actual part type to be processed at each machine may conflict due to its limited capacity and the multicriteria nature of the scheduling decisions. In this case, the actual loading part is taken according to the route priority. This latter is measured using some criterion based on the computed production rate values, the surplus performances and the order of operations for re-entrant flow.

The rest of the paper is organized as follows. Section 2 describes the continuous-flow approximation to model the discrete flow of parts in manufacturing systems. The continuous-flow control methodology is presented in section 3 . Section 4 introduces the sampling and dispatching procedure for discrete real-time scheduling of part types at shop-floor level. Section 5 illustrates the scenario and the experimental results for re-entrant and multi-product real manufacturing system. Finally, concluding remarks are given in section 6 .

\section{CONTINUOUS-FLOW DYNAMIC MODEL}

Many industrial production systems, such as semiconductor manufacturing, generate typical processes of large-scale, time-varying and stochastic systems. They involve different kinds of operation (transformation, assembly or disassembly), operate in an uncertain and unpredictable environment and manufacture a highvolume and medium-variety of products. For such manufacturing systems, continuous-flow models offer an interesting way to reduce the complexity inherent to discrete flow modelling by approximating the discrete material flows with continuous model (Brandimarte et al., 1996).

Furthermore, the manufacturing system can be viewed at the shop-floor level as a network of a finite number of machines and buffers. Thus, when considering a system composed of $N$ machines $M_{i}(i=1, \ldots, N)$ and according to the operation type (transformation, assembly and disassembly), it may be decomposed into $N$ basic production modules $P M(i)$. Each one is composed of a machine $M_{i}$ and its sets of upstream and downstream buffers denoted $B_{+}(i)$ and $B_{-}(i)$ respectively. The cardinalities of these sets distinguish the different operation types. For instance, in the case of a transformation line (Figure 1), the cardinality of the sets $B_{+}(i)$ and $B_{-}(i)$ is respectively: $\operatorname{card}\left\{B_{+}(i)\right\}=\operatorname{card}\left\{B_{-}(i)\right\}=1$ with $B_{+}(i)=\left\{B_{i-1}\right\}$ and $B_{-}$ $(i)=\left\{B_{i}\right\}$. Thus, the production module can be defined as $P M(i)=\left\{B_{i-1}, M_{i}, B_{i}\right\}$. The assembly and disassembly operations are distinguished respectively when the cardinality of the sets $B_{+}(i)$ and $B_{-}(i)$ are greater than one.

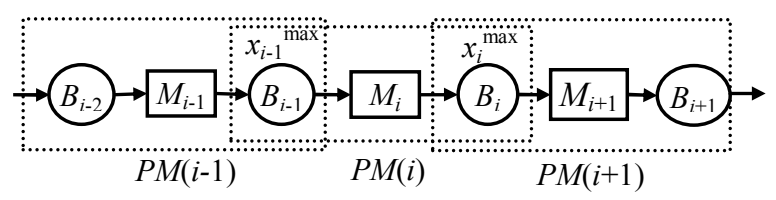

Figure 1: Production line

For the sake of simplicity, the developments are given for a single-part-type system depicted in Figure 1. The level of buffer $B_{i}$ is given by the variable $x_{i}$, collecting continuously the products coming from machine $M_{i}$ and feeding machine $M_{i+1}$. The machines are supposed unreliable. Let $\beta_{i}(t)$ be a state of the machine $M_{i}$ at time $t$ with $\beta_{i}(t)=1$ if $M_{i}$ is up and $\beta_{i}(t)=0$ otherwise. The uptimes and downtimes are assumed to be exponentially distributed with rates $\lambda_{i}$ and $\mu_{i}$ respectively. The production rate of machine $M_{i}$ at time $t$ is denoted by $u_{i}(t)$ and the required processing time, noted $\tau_{i}$, is supposed known and deterministic. When machine is up, i.e.; $\beta_{i}(t)$ $=1$, it produces continuously at some rate; which can be adjusted to any value between zero and its maximum rate $u_{i}^{\max }=1 / \tau_{i}$, i.e., $0 \leq u_{i}(t) \leq u_{i}^{\max }$. When the machine is down, i.e., $\beta_{i}(t)=0$, the production rate is zero. Thus, the increasing rate of buffer $B_{i}$ is a function of the production rate $u_{i}$ of the feeding machine $M_{i}$. The decreasing of buffer level $x_{i}$ is in relation with the processing rate $u_{i+1}$ of the downstream machine $M_{i+1}$. Therefore, by aggregating the increasing and decreasing rates, the dynamic model of the evolution of buffer level (production-flow) $x_{i}$ is given by:

$$
\dot{x}_{i}(t)=u_{i}(t)-u_{i+1}(t) \text {, with } 0 \leq x_{i}(t) \leq x_{i}^{\max }
$$

This dynamic equation represents the basis of the continuous-flow model used in simulation. The restriction in (1) concerns the inability of buffer $x_{i}$ to increase its content while the capacity bound $x_{i}^{\max }$ is reached. When considering a manufacturing composed of $N$ production modules, its dynamic is governed by $N$ differential equations according to (1).

To ensure a stable system, and thus, the existence of feasible control policies, the average system capacity is assumed to be larger than the demand rate $d$, i.e., $\left[u_{i-}\right.$ $\left.{ }^{\max } \times \lambda_{i} /\left(\lambda_{i}+\mu_{i}\right)\right]>d$ (Perkins and Kumar, 1989). In this case, let us defines the fraction of the capacity of $M_{i}$ devoted for processing at time $t$ as follow:

$r_{i}(t)=\frac{u_{i}(t)}{u_{i}^{\max }}$, with $r_{i}(t) \in[0,1]$

In this paper, $r_{i}(t)$ represents the control variable, to be defined, that adjusts the production rate between zero and its maximum. Further, in order to track the demand (requirement) at each production means, the production surplus $s_{i}$ (tracking error), defining the difference between the cumulative production (performance measure) at this means (denoted $y_{i}$ ), and the demand, is taken into account in the design of the closed loop control system. 


\section{CONTINUOUS-FLOW CONTROL METHODOLOGY}

Given a manufacturing system represented by the production-flow dynamic model (1), the control objective is to adjust the production rates, through an appropriate capacity allocation policy, in such a way to reach a predefined required production while keeping all overall performance measures within their acceptable values (Tamani et al., 2009). For this purpose, in our previous work, the continuous-flow control methodology of two levels has been developed with a set of distributed fuzzy controllers at the lower level and a supervisory controller at the higher level. This section recalls the flow control methodology principles with focuses on the supervisory control strategy.

\subsection{Distributed fuzzy control for machine's capacity allocation}

To make clear how the distributed fuzzy control strategy is designed, the basic idea is first illustrated through the elementary transformation module $P M(i)$. In our case, the control law is determined on the basis of the expert knowledge, where a fuzzy system, constituting a controller $F C(i)$, has been used. The control objective is to track the demand while keeping the upstream and downstream buffers of $M_{i}$ neither full nor empty. This is achieved by allocating an optimised machine capacity to production at each instant according the following rules:

- If the surplus level is satisfying (normal), then try to prevent starving or blocking by increasing or decreasing the production rate of the machine.

- If the surplus level indicates backlog or excess inventory, then produce respectively with the maximum or zero rate.

Thus, the input variables of the levels of upstream and downstream buffers $x_{i-1}(t), x_{i}(t)$ and the production surplus $s_{i}(t)$ of $P M(i)$ have been considered. The output variable of the controller represents a weighting factor $r_{i}(t)$ to range the production rate of $P M(i)$ between zero and its maximum $u_{i}^{\max }$. Figure 2 illustrates the fuzzy control structure $F C(i)$ for a transformation operation.

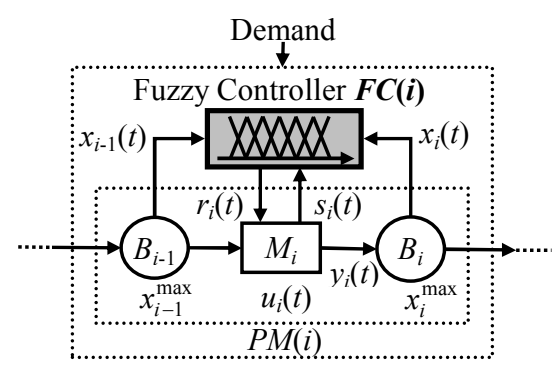

Figure 2: The fuzzy control structure

The fuzzy controller $F C(i)$ has been formalized by using a Takagi-Sugeno system (Sugeno, 1999) given by a collection of rules in the following form:

$$
\begin{aligned}
R_{i}^{\left(i_{1}, i_{2}, i_{3}\right)}: & \text { IF } x_{i-1} \text { is } X_{i-1}^{i_{1}} \text { and } x_{i} \text { is } X_{i}^{i_{2}} \text { and } s_{i} \text { is } S_{i}^{i_{3}}, \\
& \text { Then } r_{i}=\phi_{i}^{\left(i_{1}, i_{2}, i_{3}\right)}
\end{aligned}
$$

where:

- $X_{i-1}^{i_{1}}, X_{i}^{i_{2}}$ and $S_{i}^{i_{3}}$ correspond to the $i_{k}^{\text {th }}$ linguistic term of the input variables $x_{i-1}, x_{i}$ and $s_{i}$, taken respectively from the sets $X_{i-1}=X_{i}=\{$ Empty, Almost Empty, Normal, Almost Full, Full $\}$ and $S_{i}=\{$ Back$\log$, Normal, Inventory\}.

- $\phi_{i}^{\left(i_{1}, i_{2}, i_{3}\right)}$ is the real value involved in the rule conclusion indexed by $\left(i_{1}, i_{2}, i_{3}\right)$ that gives the fraction of capacity devoted to processing.

Figure 3 shows the response surfaces of the fuzzy controller where the effect of the buffer levels on the processing rate for some values of the surplus is investigated. The universe of discourse of the buffer levels $x_{i-1}$ and $x_{i}$ are normalized with regard to the buffer sizes $x_{i-}$ ${ }_{1}^{\max }$ and $x_{i}^{\max }$ respectively in order to take values within $[0,1]$ (Figure 3).

The complete rulebase for a fuzzy controller of a transformation module is given in (Tamani et al., 2009). In the case of assembly and disassembly modules, the fuzzy controller is designed similarly by tacking into account more than one upstream buffer level and more than one downstream buffer level respectively as input variables in premises of rules of the form (3). The rule bases are then built in such a way to synchronize the operations with the objective of balancing the system load.
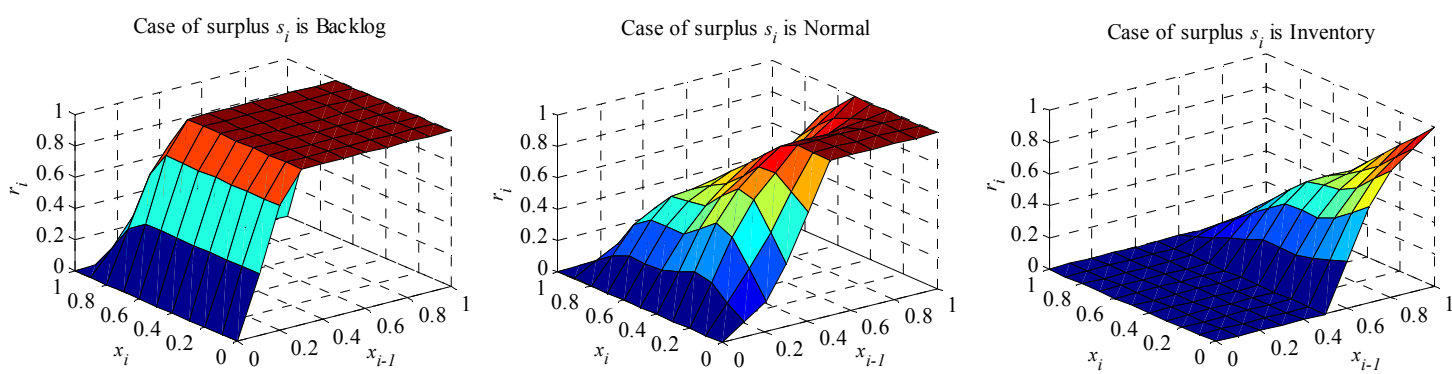

Figure 3: Response surfaces of the fuzzy controller 
Finally, when considering a general manufacturing system composed of $N$ modules, the fuzzy control design detailed above has been deployed for each ones, which leads to a distributed fuzzy control (DFC) structure. The major advantage of this control architecture resides in its modularity and distributivity that enhance the flexibility of the system and make easily the implementation phases in complex manufacturing systems.

\subsection{Supervisory based fuzzy arithmetic interval}

In fully distributed control systems, global optimization is hard to obtain due to the difficulty of proving that a sufficient level of performance can be attained. This difficulty is mainly due to the "myopic behaviour" of distributed control systems. In order to maintain a certain performance level, it is frequently necessary to deal with myopic behaviour by defining a kind of "global optimizing mechanism" (GOM) (Trentesaux, 2009).

There are several ways to integrate GOM into distributed control systems. Common solutions are to ensure that local decisions are made in consideration of global criteria, or to impose global specifications within which global performance level must be maintained. The last idea has been considered in order to integrate GOM through a supervisory controller. Indeed, given a set of performance indicators $\mathrm{P}=\left\{P_{1}, \ldots, P_{L}\right\}$ with associated objectives $\mathrm{P}^{\text {obj }}=\left\{P_{1}{ }^{\text {obj }}, \ldots, P_{L}{ }^{\text {obj }}\right\}$, the supervisory controller aims at reinforcing the local control action through an additive component in order to compensate the deviations of performance measures from their objectives. The key idea of the supervision function resides in: (i) the fuzzy intervals representation of the objectives and (ii) the combination mechanism based on the fuzzy interval arithmetic.

For the first point, a trapezoidal fuzzy interval, denoted by $P_{l}^{\text {obj }}$, has been used to represent the objective associated to the performance indicator $P_{l}$ as illustrated in Figure 4. The shape of the fuzzy interval is specified by two functions that link the support and the kernel values according to the vertical dimension. These functions, denoted by $\left(P_{l}^{\mathrm{obj}}\right)^{-}$(the increasing part in Figure 4) and $\left(P_{l}^{\mathrm{obj}}\right)^{+}$(the decreasing part in Figure 4), are respectively called the left and right profiles (Klir, 1997). In the case of trapezoidal shape, they are defined by:

$$
\left\{\begin{array}{l}
\left(P_{l}^{\mathrm{obj}}\right)^{-}(\alpha)=(1-\alpha) \cdot a_{l}^{\mathrm{obj}}+\alpha \cdot b_{l}^{\mathrm{obj}} \\
\left(P_{l}^{\mathrm{obj}}\right)^{+}(\alpha)=(1-\alpha) \cdot d_{l}^{\mathrm{obj}}+\alpha \cdot c_{l}^{\mathrm{obj}}
\end{array}\right.
$$

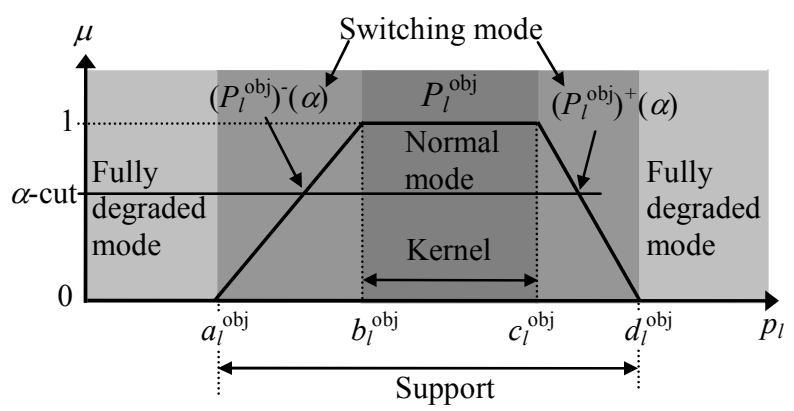

Figure 4: Trapezoidal fuzzy interval representation
According to the trapezoidal representation of the objective, the satisfaction may be total when the performance indicator evolves within the kernel (normal mode), not satisfactory at all if it is outside the support (fully degraded mode), and not satisfactory with different degrees when it is limited by the left or right profiles (switching mode) (Figure 4). These different situations distinguish the operating modes of the production system.

For the second point, given the fuzzy intervals of the objectives, the principle of the supervision mechanism is summarized on the following three steps:

- Step 1: Combine the objectives $\mathrm{P}^{\text {obj }}=\left\{P_{1}{ }^{\text {obj }}, \ldots\right.$, $\left.P_{L}{ }^{\text {obj }}\right\}$ through an uncertain operator $\Psi$, since they are defined by fuzzy intervals. The combined objective is a fuzzy interval denoted $\mathrm{P}_{\mathrm{T}}^{\text {obj }}$.

- Step 2: Combine the performance indicator measures $\mathrm{P}=\left\{P_{1}, \ldots, P_{L}\right\}$ using the precise version of the operator $\Psi$, denoted $\psi$. The combined measure is denoted $\mathrm{P}_{\mathrm{T}}$.

- Step 3: Evaluate the resulted precise measure $\mathrm{P}_{\mathrm{T}}$ with regard to the combined fuzzy objective $\mathrm{P}_{\mathrm{T}}^{\text {obj }}$. The result represents the satisfaction degree of the combined objective (the $\alpha$-cut) which indicates the current operating mode.

At the first step, the arithmetic operations on fuzzy intervals are used according to the profiles representation (4). In this case, the uncertain operator can be implemented (Boukezzoula et al., 2007). For instance, when using the weighted mean operator, the resulted fuzzy interval is expressed as follows:

$$
\mathrm{P}_{\mathrm{T}}^{\mathrm{obj}}=\Psi\left[P_{1}^{\mathrm{obj}}(\alpha), \ldots, P_{L}^{\mathrm{obj}}(\alpha)\right]=\sum_{l=1, \ldots, L}^{\oplus} w_{l} \cdot P_{l}^{\mathrm{obj}}(\alpha)
$$

where $\Sigma^{\oplus}$ is the fuzzy addition between fuzzy intervals such that: $\left(P_{l}^{\text {obj }} \oplus P_{k}^{\text {obj }}\right)(\alpha)=\left[\left(P_{l}^{\text {obj }}\right)^{-}(\alpha)+\left(P_{k}^{\text {obj }}\right)^{-}(\alpha)\right.$, $\left.\left(P_{l}^{\text {obj }}\right)^{+}(\alpha)+\left(P_{k}^{\text {obj }}\right)^{+}(\alpha)\right]$.

The second step is performed in the same way by considering the precise performance indicator measures according to the precise operator (Dubois et al., 2004). Finally, at the third step, the resulted satisfaction degree (the $\alpha$-cut) is used to determine the additive component (supervisory control action), denoted $r_{s_{i}}$, under the constraint of the local control $r_{c_{i}}$. Figure 5 illustrates the synopsis of the supervision principle

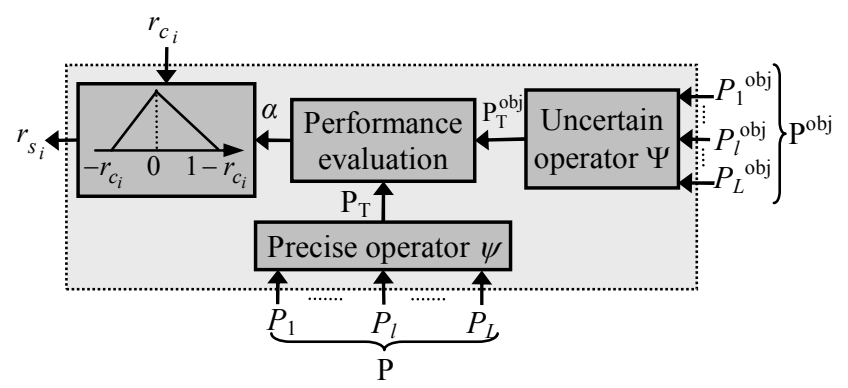

Figure 5: Synopsis of the supervision principle 
The different values of $r_{S_{i}}$ are encapsulated within a triangular fuzzy interval $R_{s_{i}}$ with the support $R_{s_{i}}(0)=\left[-r_{c_{i}}, 1-r_{c_{i}}\right]$ and the kernel $R_{s_{i}}(1)=0$. As shown in Figure 6 when the combined objective is still trapezoidal, it can be observed that the switching between the operating modes is gradual, which reduces the chattering phenomenon that may occur around the limits of conventional interval, and thus involves more stable performances. The limit values of the supervisory control action consist to allocate the maximum remaining capacity $\left(1-r_{c_{i}}\right)$ or to stop the production $\left(-r_{c_{i}}\right)$ of the module $P M(i)$. The intermediate values allow weighting the support bounds of $R_{s_{i}}$ according to the satisfaction degree (the $\alpha$-cut) of the combined overall objective $\mathrm{P}_{\mathrm{T}}^{\mathrm{obj}}$ (Figure 6).

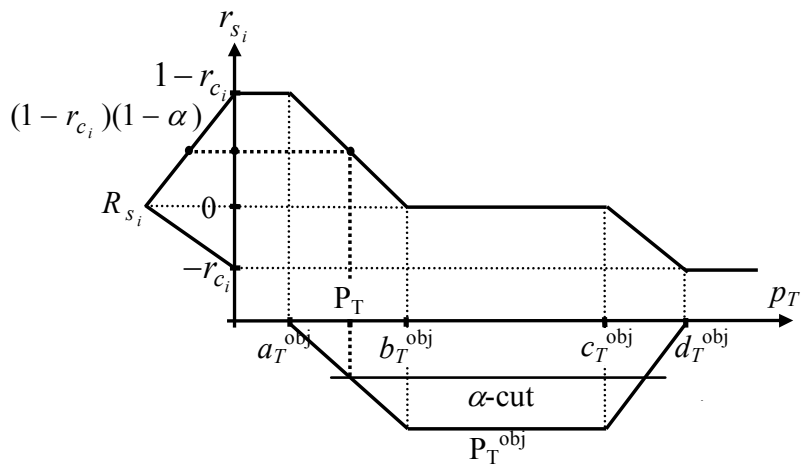

Figure 6: The evolution of the supervisory control

For practical implementation, the supervisory control is determined according to the following statements:

- If $\mathrm{P}_{\mathrm{T}}$ evolves within the kernel of $\mathrm{P}_{\mathrm{T}}{ }^{\text {obj }}$, the system behaviour is in normal mode. This means that the satisfaction degree of the objective is total $(\alpha=1)$. In this case, the supervisor does not provide additive component $\left(r_{s_{i}}(t)=0\right)$.

- If $\mathrm{P}_{\mathrm{T}}$ evolves outside the support of $\mathrm{P}_{\mathrm{T}}{ }^{\text {obj }}$, a fully degraded operating mode is detected. The objective in this case is totally unsatisfied $(\alpha=0)$, and the supervisory action is given by:

$r_{s_{i}}(t)=\Delta-r_{c_{i}}(t)$ with $\Delta= \begin{cases}1 & \text {,if } \mathrm{P}_{\mathrm{T}} \leq a_{T}^{\text {obj }} \\ 0 & \text {,if } \mathrm{P}_{\mathrm{T}} \geq d_{T}^{\text {obj }}\end{cases}$

It consists in either allocate the maximum remaining capacity $(\Delta=1)$ or stop the productivity of the module $(\Delta=0)$.

- If $\mathrm{P}_{\mathrm{T}}$ evolves in the switching modes, the corresponding $\alpha$-cut of the fuzzy interval $\mathrm{P}_{\mathrm{T}}{ }^{\text {obj }}$ is used to determine the supervisory control. Indeed, whether $\mathrm{P}_{\mathrm{T}}$ evolves on the left or right profile, the $\alpha$-cut level is given by the reverse of the corresponding profile function. That is, in one hand, when $\mathrm{P}_{\mathrm{T}}$ evolves on the left profile, the supervisory control is given as:

$r_{s_{i}}(t)=(1-\alpha) \cdot\left(1-r_{c_{i}}(t)\right)$ with $\alpha=\left(\left(\mathrm{P}_{\mathrm{T}}^{\mathrm{obj}}\right)^{-}\left(\mathrm{P}_{\mathrm{T}}\right)\right)^{-1}$

In this case, the action attempts to allocate a fraction of the remaining capacity. On the other hand, when $P_{l}$ evolves on the right profile, the supervisory action attempts to reduce the productivity of the controlled module as follows:

$r_{s_{i}}(t)=(1-\alpha) \cdot\left(-r_{c_{i}}(t)\right)$ with $\alpha=\left(\left(\mathrm{P}_{\mathrm{T}}^{\mathrm{obj}}\right)^{+}\left(\mathrm{P}_{\mathrm{T}}\right)\right)^{-1}$

The functions (7) and (8) represent respectively the right and left profiles of a triangular fuzzy interval $R_{s_{i}}$ of the supervisory control domain (Figure 6).

Finally, according to the local control given by the fuzzy controller and the supervisory control, the production rate is adjusted as follow:

$u_{i}(t)=\left(r_{c_{i}}(t)+r_{s_{i}}(t)\right) \cdot u_{i}^{\max }=r_{i}(t) \cdot u_{i}^{\max }$

The proposed distributed-supervised control structure is illustrated in Figure 7 for the case of transfer line composed by $N$ transformation modules.

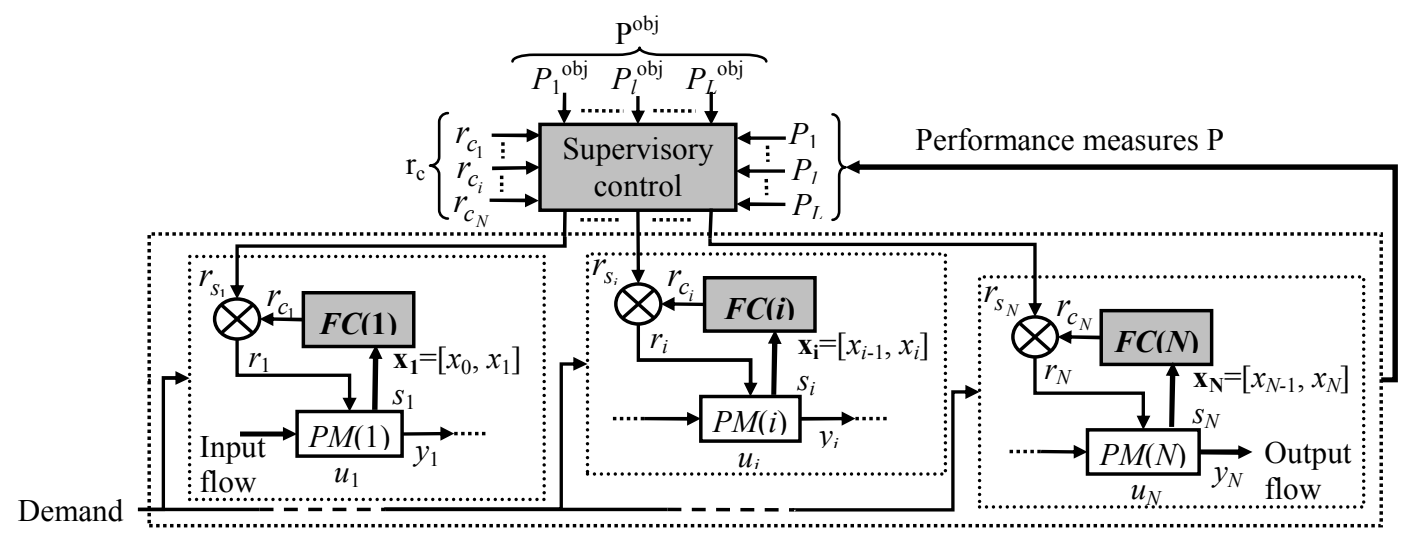

Figure 7: The continuous-flow control architecture 


\section{DISCRETE REAL-TIME SCHEDULING METHODOLOGY}

Conventional formulation of the scheduling problem consists in the optimisation of some criterion, such as the makespan and total production cost, under time and resource constraints. In the previous section, the production rates (9) are approximated by a continuous expression while the production operations are of discrete nature. Therefore, considering the criterion of makespan and production cost minimisation, the scheduling problem involves two types of decisions at this level:

- to determine the loading times of actual parts and

- to resolve the conflicts in the case of multiple-parttype systems.

For the first decision, a dispatching policy has to be used in order to determine the loading times of actual parts. More precisely, the obtained continuous-time control variables have to be translated into a dispatching time series through a sampling procedure. Indeed, since the machine operation frequency is equivalent to the time between two successive machine loads, at a certain time, the sampled value is held constant during a time interval equal to its reverse. The holding period includes the operation and the idle times. Thus, the continuous time production rate is translated to a piece-wise constant function as shown in Figure 8.

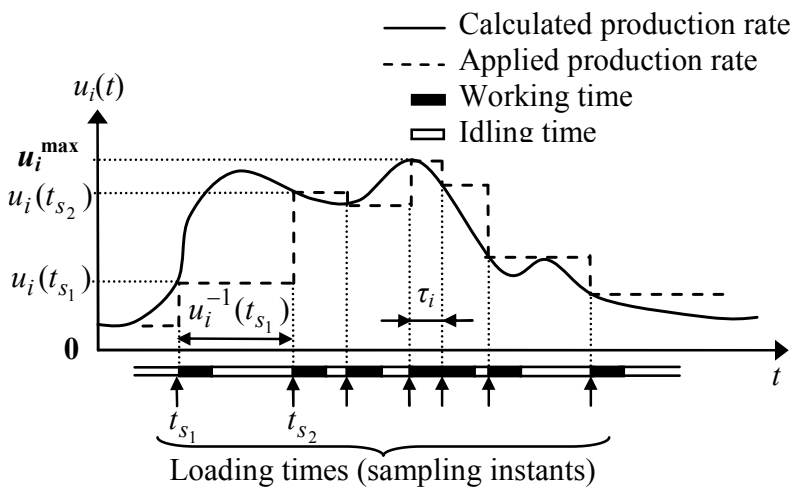

Figure 8: Continuous production rate discretisation

Using this definition, as the production rate evolves between 0 and $u_{i}^{\max }$, the lower bound correspond to an infinite idling time (no production) while the upper bound corresponds to the operation time (no idle time). For practical use, in order to limit the idle period when the production rate is too low, the lower bound is chosen equal to $50 \%$ of its maximum.

For the case of a multiple-part-type system, a machine $M_{i}$ may operates on different part types $j$ such that $j \in Q(i)$, where $Q(i)$ is the set of part types to be processed on $M_{i}$ and its cardinality is equal to $J(i)$. Each of them may involves $K_{i j}\left(k=1, \ldots, K_{i j}\right)$ different operations (case of re-entrant flow if $K_{i j}>1$ ). In this case, the original machine $M_{i}$ is virtually divided into $N(i)=\sum_{\{j j \in \in(i)\}} K_{i j}$ single-part-type sub-machines $m_{i j k}$. Set-up times are assumed to be insignificant. Only one submachine is allowed to work at a time. Thus, for the second decision, in order to deal with conflicts in the scheduling problem, the actual parts to be processed at each machine are determined according to a decision function based on some criterion representing the route priority measurement. The derivation of the criterion value is based on the control input values; the surplus performances (local and final) and the order of the operation in the case of reentrant flow. The part to be loaded is the one with the largest criterion value. The proposed criterion value for each submachine $m_{i j k}$ of a certain multiple-part-type machine $M_{i}$ is given by the following weighting sum:

$J_{i j k}=\sum_{l=1}^{4} \pi_{l} \cdot g\left(c_{i j k}^{l}\right)$

where:

- $\quad c_{i j k}^{1}$ is the sampled value of the computed production rate $\hat{u}_{i j k}$ of the submachine $m_{i j k}$,

- $\quad c_{i j k}^{2}$ is its corresponding local surplus such that $c^{2}{ }_{i j k}$ $=\max \left\{0,-s_{i j k}\right\}$,

- $\quad c_{i j k}^{3}$ is the finished surplus level $s_{O(j)}$ of the part-type $j$, with $O(j)$ is the last submachine of its route,

- $\quad c_{i j k}^{4}$ is the order $k$ in which the part of type $j$ visits the machine $M_{i}$.

In the criterion definition above, $g($.$) is a positive$ monotonically increasing non-linear function, with $g(0)$ $=0$ and $g\left(c_{i j k}^{l}\right)=1$ for $c_{i j k}^{l} \rightarrow \infty$. This function can be closely approximated by sigmoidals of the form: $g\left(c_{i j k}^{l}\right)=$ $1 /\left(1+\exp \left(-c_{i j k}^{l}\right)\right)$ (Rovithakis et al., 1999). According to the measures of $c_{i j k}^{l}(l=1, \ldots, 4)$, this function gives the maximum value for the route (submachine) which presents the highest calculated production rate, the larger backlog (negative local and final surpluses) and the latest operation in the case of re-entrant flow. The values of $c^{1}{ }_{i j k}, c^{2}{ }_{i j k}$ and $c^{4}{ }_{i j k}$ lead to a criterion with a local scope, while $c^{3}{ }_{i j k}$ introduces global insight of the state of the actual route. The parameters $\pi_{l}$ are the weighting factors to be chosen according to the importance of each element $c_{i j k}^{l}$. The following algorithm summarizes a practical implementation of the discrete dispatching procedure:

\section{Inputs}

$u_{i} \in \boldsymbol{R}^{N(i)}, s_{i} \in \boldsymbol{Z}^{N(i)}, s_{O_{i}} \in \boldsymbol{Z}^{J(i)}$ with $O_{i}=\{O(j) \mid j=1, \ldots$, $J(i)\}$.

\section{Outputs}

The selected submachine $m_{i j k}$ with its discrete production rate $\hat{u}_{i j k}$, loading time $t_{s_{n}}^{*}$ and holding time interval $\hat{u}_{i j k}^{-1}$.

\section{Begin}

(1) For all not idle submachines

Calculate $J_{i j k}$ according to (10).

Select the submachine $m_{i j k}$ having the highest $J_{i j k}$. 


\section{Endfor}

(2) The production rate $u_{i j k}$ of the selected submachine is sampled at a time $t_{s_{n}}^{*}(n=1,2 \ldots)$ corresponding to the loading instant. A time interval equal to the inverse of the sample is computed ( $\hat{u}_{i j k}^{-1}$ according to $\left.\hat{u}_{i j k}=u_{i j k}\left(t_{s_{n}}^{*}\right)\right)$. The values of the production rates evolving during the holding time are ignored.

(3) As soon as the time interval is competed, a new sample of the production rate is considered and the process is repeated (go to step 1).

\section{End}

Figure 9 summarises the production-flow scheduling methodology in which the flow control level interacts with the shop-floor one over the discretisation procedure of the production rates and the continuous approximation of the discrete flow of parts.

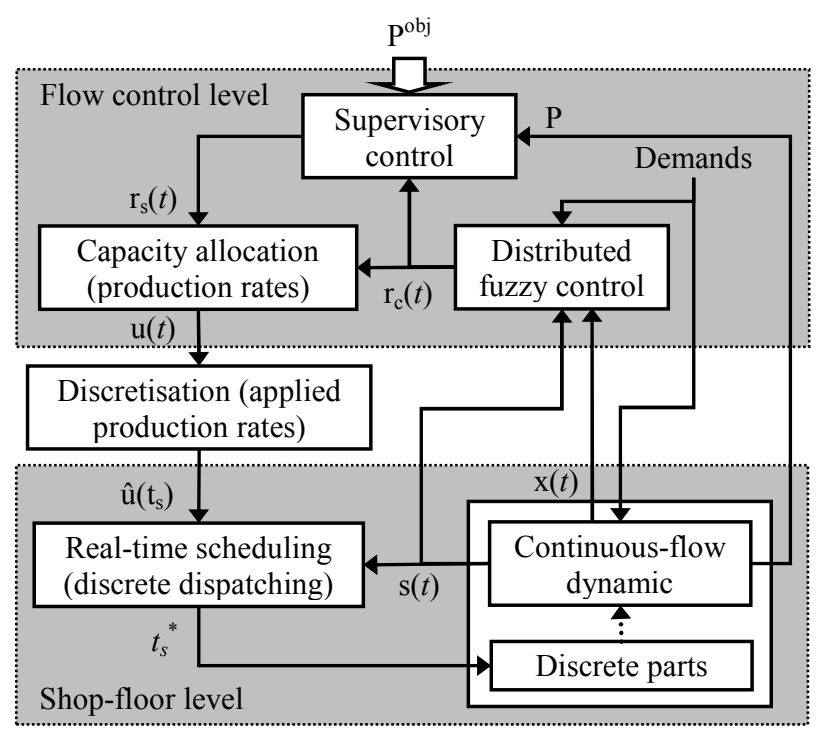

Figure 9: The production-flow scheduling methodology

\section{SCHEDULING METHODOLOGY APPLICATION}

In this section, the developed scheduling methodology is illustrated through a simulation study performed on a realistic example of a manufacturing cell taken from (Rovithakis et al., 1999). Comparisons with the results reported herein of the existing scheduling policies and those obtained with the first in first out (FIFO) policy are performed. Specifically, clear a fraction (CAF), clear largest buffer (CLB) (Perkins and Kumar, 1989), and the dynamic neural network scheduler (DNN) developed in (Rovithakis et al., 1999), have been employed.

The proposed scheduling methodology is implemented by means of Matlab/Simulink and Floulib toolbox (Foulloy et al., 2006) (available at http://www.listic.univ-savoie.org), while the FIFO policy is tested through discrete event simulator with the help of the Apollo platform (Habchi and Berchet, 2003).

\subsection{Manufacturing cell description and simulation results}

The considered system consists of five machines and produces five different part types. Due to one assembly process, six routes are defined (Table 1). The routes 2, 3, 4 and 5 lead to finished products. The routes 1 and 6 lead to the part types to be assembled on machine 5. The table elements show the order in which every product visits the machines. A production demand of 20 parts for each of the 5 part types has to be achieved. For simplicity, the operation times for all products on the same machine are assumed to be equal. The machine operation times are taken equal to 5, 6, 5, 4 and 3 time units respectively.

The number of operations for the second machine is equal to 7 instead 6 , since it serves part type 1 twice (reentrant flow). The same holds for machine $M_{3}$, where part type 4 is also served twice. Furthermore, raw materials arrive in the cell at a rate of 0.03 part per time unit, implying that for each route a raw material arrives every 34 time units, and is stored in the buffer of raw materials. All buffers in the cell are considered to have a capacity of 15 parts. Raw materials arrive to the system at the specified rate, for so long as the production of the respective product is not complete, and the buffer storing raw materials has not reached its capacity.

\begin{tabular}{|l|l|l|l|l|l|}
\hline \multicolumn{5}{|l|}{ Machine } \\
\hline Route & $M_{1}$ & $M_{2}$ & $M_{3}$ & $M_{4}$ & $M_{5}$ \\
\hline 1 & & 2,4 & 3 & 1 & 5 \\
\hline 2 & 1 & 2 & & & \\
\hline 3 & & 1 & 2 & & 3 \\
\hline 4 & 2 & 5 & 1,4 & 3 & \\
\hline 5 & 3 & 2 & & 1 & \\
\hline 6 & & 2 & 1 & 3 & 4 \\
\hline
\end{tabular}

Table 1: Part types routes

Based on the workload of the cell bottleneck machine, i.e. machine $M_{2}$; the authors in (Rovithakis et al., 1999) define a lower bound for the achievement of the production demand (makespan) which serves as the reference for comparison purposes. Specifically, the machine $M_{2}$ ( 7 submachines) must process 20 parts requiring $7 \times 20 \times 6=840$ time units. As the first raw material arrives in the cell at time 34, a lower bound of 874 time units has been derived.

In order to evaluate the effect of the supervision, the proposed methodology is simulated in both cases: without supervision (distributed fuzzy control - DFC) and with supervisory control (supervisory fuzzy control SFC). When integrating the supervisory control, the overall performance indicators of the average and the instantaneous finished surplus, and the total production cost are used. This latter is given by:

$$
\text { Total Cost }=c_{w i p} \cdot \overline{W I P}+c_{i n v} \cdot \overline{I N V}+c_{b c k} \cdot \overline{B C K}+c_{l t} \cdot \overline{L T}
$$

The first two terms of (11) represent the cost measures of storing parts in buffers. Specifically, measures for the 
work-in-process and inventory costs are provided by means of the average integral of the intermediate and output buffers respectively. That is: $\overline{W I P}=1 /\left(T \times N_{i n}\right) \sum_{\left\{i, j, k \mid m_{i j k} \neq O(j)\right\}} \int_{0}^{T} x_{i j k}(t) d t \quad$ and $\overline{I N V}=1 /\left(T \times N_{\text {out }}\right) \sum_{j=1}^{N_{\text {out }}} \int_{0}^{T} x_{O(j)}(t) d t$ with $N_{\text {in }}=16, N_{\text {out }}$ $=5$ are the number of the intermediate and output buffers in the cell and $T$ is the production makespan (i.e., the time period elapsing until the production goal is achieved for all products types).

The two last terms of (11) are concerned with the average backlogging costs (i.e., the cost resulting from the delay in achieving production targets) and the average lead time costs (i.e., the average of the time elapsing between the input of a raw material in the cell and the output of the respective finished product). Specifically, the employed average backlog is defined as follows: $\overline{B C K}=1 /\left(T \times N_{\text {out }}\right) \sum_{j=1}^{N_{\text {out }}} \int_{0}^{T}\left(\right.$ In $\left._{j}(t)-O u t_{j}(t)\right) d t$ with $\operatorname{In}_{j}(t), \operatorname{Out}_{j}(t)$ the number of parts of type $j$ that have entered and exit the cell until time $t$.

The cost units $c_{w i p}, c_{i n v}, c_{b c k}, c_{l t}$ for all the performance measures in (11) are taken equal to 1 for simplicity. The measure of the total cost in the supervisor is chosen as the reverse of (11). The associated objectives, expressed by fuzzy intervals through the profile functions (4), are fixed, for the surplus performances, as:
$P_{1}^{\text {obj }}=P_{2}^{\text {obj }}=[-3+2 \alpha, 3-2 \alpha]$, and for the total production cost performance as: $P_{3}^{\text {obj }}=[0.01+0.09 \alpha, 1.1-0.1 \alpha]$. When using the arithmetic mean operator (5), the resulted combined interval is: $\quad \mathrm{P}_{\mathrm{T}}^{\text {obj }}=[-1.996+1.363 \alpha, 2.366-1.366 \alpha]$. The parameters $\pi_{l}$ of the criterion (10) are taken respectively equal to $0.4,0.25,0.25,0.1$.

The obtained results are compared to the conventional FIFO strategy and those provided in (Rovithakis et $a l ., 1999)$, and are summarized in Table 2 for the case of reliable machines. In this case, the machine utilisation rates are given in Table 3, while Figure 10 presents the comparison of the evolution of the finished cumulative production for each part type obtained by the schedulers FSC, DFC and FIFO.

Table 4 shows, in terms of average of ten simulation runs, the results for the case where the machines are unreliable. In this case, the repair rates are all equal to $\mu_{i}$ $=0.5$ and the failure rates are $\lambda_{i}=0.1$.

\begin{tabular}{|l|l|l|l|l|l|}
\hline & \multicolumn{5}{|l|}{ Machine utilisation rates (\%) } \\
\hline Methodology & $M_{1}$ & $M_{2}$ & $M_{3}$ & $M_{4}$ & $M_{5}$ \\
\hline SFC & 40.05 & $\mathbf{9 6 . 1 1}$ & 65.22 & 43.02 & 13.73 \\
\hline DFC & 34.76 & 83.42 & 56.11 & 38.13 & 11.92 \\
\hline FIFO & 34.21 & $\mathbf{9 5 . 7 8}$ & 57.01 & 36.49 & 13.68 \\
\hline
\end{tabular}

Table 3: Machine utilisation rates without failures

\begin{tabular}{|l|l|l|l|l|l|l|}
\hline Methodology & Makespan & Avg. WIP & Avg. inventory & Avg. backlog & Avg. lead time & Total cost \\
\hline SFC & $\mathbf{8 7 4}$ & 0.894 & 9.393 & 2.978 & 65.46 & $\mathbf{7 8 . 7 3}$ \\
\hline DFC & 1007 & 1.636 & 8.516 & 5.807 & 188.1 & 204.06 \\
\hline FIFO & 877 & 1.016 & 10.045 & 5.604 & 74.68 & 91.345 \\
\hline DNN & 963 & 0.506 & 8.17 & 0.00466 & 215.96 & 224.64 \\
\hline CAF & 1044 & 1.347 & 10.848 & 0.00262 & 142.763 & 154.96 \\
\hline CLB & 1083 & 1.149 & 11.468 & 0.00214 & 125.72 & 138.34 \\
\hline
\end{tabular}

Table 2: Simulation results without machine failures

\begin{tabular}{|l|l|l|l|l|l|l|l|}
\hline \multicolumn{2}{|l|}{ Methodology } & Makespan & Avg. WIP & Avg. inventory & Avg. backlog & Avg. lead time & Total cost \\
\hline \multirow{4}{*}{ SFC } & Mean & $\mathbf{9 6 5 . 7}$ & 1.239 & 9.545 & 4.17 & 91.583 & 106.54 \\
\cline { 2 - 8 } & Std & 16.3 & 0.067 & 0.113 & 0.238 & 6.676 & 6.901 \\
\cline { 2 - 8 } & Max & 998 & 1.321 & 9.721 & 4.463 & 101.9 & 116.9 \\
\hline \multirow{4}{*}{ DFC } & Mean & 1076.6 & 1.795 & 8.649 & 6.74 & 217.56 & 234.75 \\
\cline { 2 - 8 } & Std & 11.157 & 0.039 & 0.058 & 0.156 & 7.056 & 7.253 \\
\cline { 2 - 8 } & Max & 1107 & 1.878 & 8.728 & 7.11 & 231.9 & 249.6 \\
\hline \multirow{3}{*}{ FIFO } & Mean & 1004.1 & 1.455 & 10.018 & 6.1807 & 120 & 137.64 \\
\cline { 2 - 8 } & Std & 14.216 & 0.068 & 0.0063 & 0.1856 & 6.975 & 7.219 \\
\cline { 2 - 8 } & Max & 1030 & 1.565 & 10.026 & 6.475 & 131.92 & 149.95 \\
\hline
\end{tabular}

Std: standard deviation

Table 4: Simulation results with machine failures 

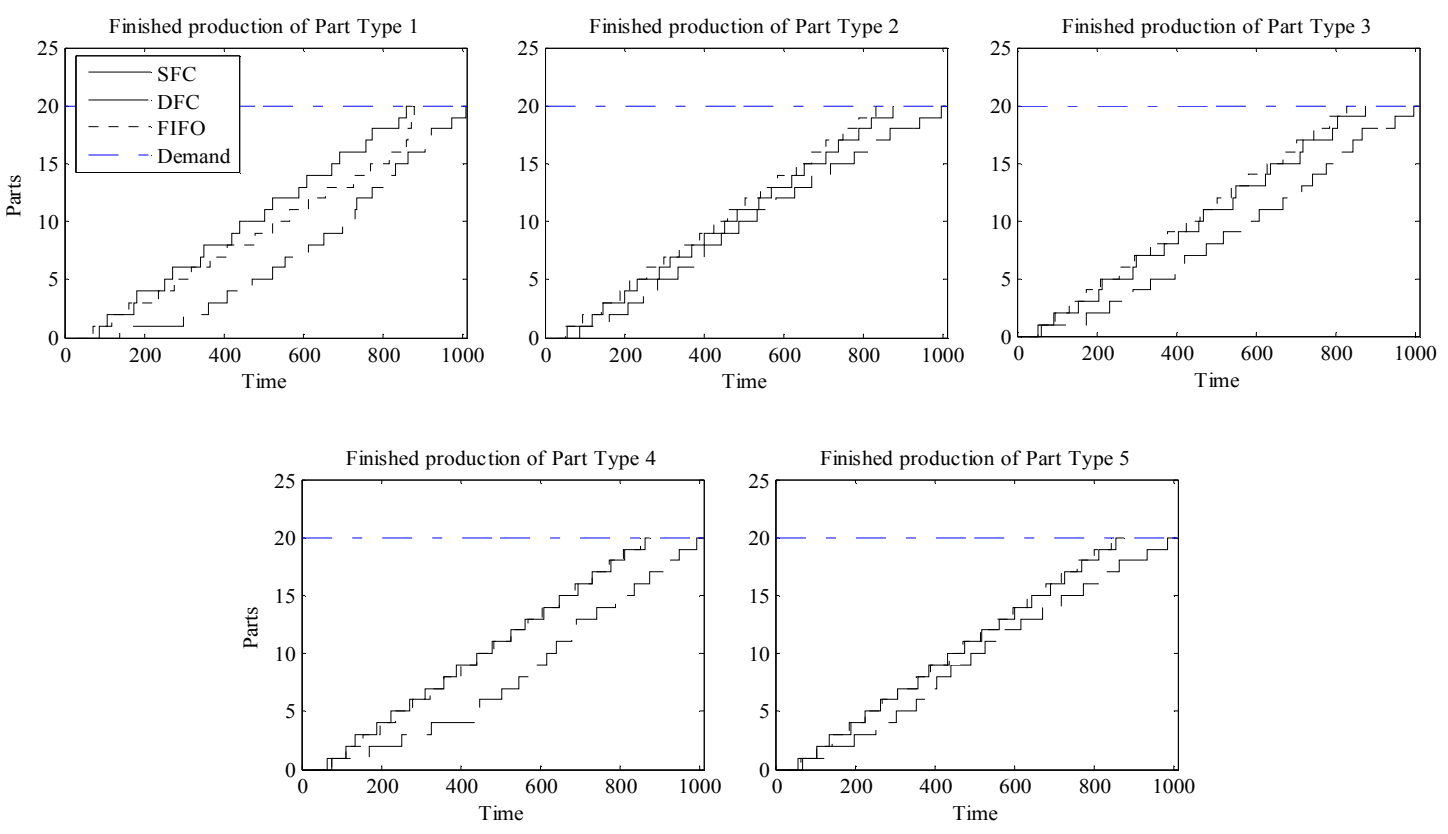

Figure 10: The evolution of finished production of each part type

\subsection{Discussion}

Based on the obtained results, both the SFC and the FIFO emerge as schedulers guaranteeing accurate achievement of production. Indeed, the SFC methodology achieves the demand with the exact calculated lower bound in the case of reliable machines (Table 2, in bold), and with a deviation of $10.49 \%$ from the lower bound when unreliable machines are considered (Table 4). The conventional FIFO scheduler achieves the production goal in 877 time units for reliable manufacturing cell thus obtaining very short deviation from the lower bound $(0.34 \%$, Table 2$)$. In presence of machine failures, the deviation observed by FIFO scheduler is equal to $14.88 \%$ (Table 4). The utilisation rate of the bottleneck machine $\left(M_{2}\right)$ in the case of SFC and FIFO methodologies, which is approximately $96 \%$, is improved in comparison to the rate reached with a DFC methodology (Table 3). This behaviour was expected since the supervisory goals are to maintain the overall production objectives within their specified domain limits by allocating the remaining production capacity when backlogs are detected.

The four last columns of Tables 2 and 4 represent average cost measures of storing parts, backlogging and lead times for the reliable and unreliable manufacturing cell respectively. The last column provides the total production cost in both cases. It can be observed that the SFC methodology achieves acceptable WIP, inventory and lead time costs due to the overall faster fulfilment of the demand. Conversely, the backlog cost is relatively worse than the values obtained by the conventional (CLB, CAF) and DNN schedulers, while it is better when compared to FIFO and DFC. These results are due to the fact that the buffers storage performances (positive surplus - WIP and inventory) and the backlog (negative surplus) are conflicting and the proposed supervisory control attempts to achieve the best compromise which is obtained in term of total production cost (Table 2, in bold).

Finally, the derived results are very promising, since the decision method is flexible when considered in conjunction with the real-time property and the multiple production objectives. This may help to cope with the conflicting control objectives and make the scheduling process more efficient in the real-time control of manufacturing systems.

\section{CONCLUSION}

In this paper, the potential application of the productionflow control for discrete scheduling of a manufacturing cell is investigated. The production-flow control methodology, initially developed in (Tamani et al., 2009), consists of distributed fuzzy controllers which are supervised by a higher level of decision-making. The objective is to allocate the limited production capacity by adjusting the machine processing rates such that the specified global performances are guaranteed. The supervisory mechanism, which is based on arithmetic fuzzy interval, provides additive components to the local controllers when degraded operating modes are detected. The supervisor is built according to the satisfaction degree of the different and possible conflicting objectives quantified by fuzzy intervals.

At the shop-floor level, the scheduling problem is addressed in two steps. The first step performs the transition from a computed continuous control to a discrete dispatching control through a sampling procedure. The principle consists to transform the calculated production rates to time intervals by taking their inverse. The second 
step deals with the conflicts of multiple routes by using some criterion representing a measure of the priority. The proposed criterion is based on the production rates, the surplus performances and the order in which the products visit the machine. Comparisons through simulations show that the proposed methodology achieves the production in the shortest time with regard to conventional schedulers.

The only uncertainties considered in this paper are the overall objectives quantification and the machine failures. An important open issue is the robustness of the methodology when other forms of uncertainty are present, such as random arrival, service, setup times etc. Another interesting extension would be the integration of the diagnosis function in the control level in order to schedule the maintenance activities in the shop-floor level.

\section{REFERENCES}

Akyol, D.E. and Bayhan, G.M., 2007. A review on evolution of production scheduling with neural networks. Computers \& Industrial Engineering, $53(1)$, p. $95-122$.

Boukezzoula, R., Galichet, S. and Foulloy, L., 2007. MIN and MAX Operators for Fuzzy Intervals and their Potential Use in Aggregation Operators. IEEE Transactions on Fuzzy Systems, 15 (6), $1135-1144$.

Brandimarte, P., Sharifnia, A. and Von Turkovich, B.F., 1996. Continuous Flow Models of Manufacturing Systems: a Review. CIRP Annals - Manufacturing Technology, 45 (1), 2441 - 444.

Brucher, P., Jurisch, B. and Sievers, B., 1994. A branch and bound algorithm for the job-shop scheduling problem. Discrete Applied Mathematics, 49 $(1-3), 107-127$.

Chan, F.T.S., Chan, H.K., Lau, H.C.W. and Ip, R.W.L., 2003. Analysis of dynamic dispatching rules for a flexible manufacturing system. Journal of Materials Processing Technology, 138 (1 - 3), $325-331$.

Cho, S. and Prabhu,V.V., 2000. Distributed adaptive control of production scheduling and machine capacity. Journal of Manufacturing Systems, $26,65-74$

Dang, T.-T. and Frankovic, B., 2002. Agent-based scheduling in production systems. International Journal of Production Research, 40 (15), 3669 -3679 .

Dubois, D. and Prade, H., 2004. On the use of aggregation operations in information fusion processes. Fuzzy Sets Systems, 142 (1), 143 - 161.

Dubois, D., Fargier, H. and Fortemps, P., 2003. Fuzzy scheduling: Modelling flexible constraints vs. coping with incomplete knowledge. European
Journal of Operational Research, 147 (2), 231 -252 .

Foulloy, L., Boukezzoula, R. and Galichet, S., 2006. An educational tools for fuzzy control. IEEE Transactions on Fuzzy Systems, 14 (2), 217 221.

Gershwin, S.B., 2000. Design and Operation of Manufacturing Systems - The Control Point Policy. IIE Transactions, 32 (2), 891 - 906.

Grabot, B., 2001. Artificial intelligence and soft computing for planning and scheduling: how to efficiently solve more realistic problems. Engineering Applications of Artificial Intelligence, 14 (3), $265-267$.

Habchi, G. and Berchet, C., 2003. A model for manufacturing systems simulation with control dimension. Simulation Modelling Practice and Theory, $11,21-44$.

Klir, G.J., 1997. Fuzzy arithmetic with requisite constraints. Fuzzy Sets and Systems, 91 (2), 165 175.

Pinedo, M.L., 2002. Scheduling: theory, algorithms, and systems. NJ: Prentice-Hall.

Perkins, J. and Kumar, P.R., 1989. Stable Distributed Real-Time Scheduling of Flexible Manufacturing / Assembly / Disassembly Systems. IEEE Transactions on Automatic Control, 34 (2), 139 -148 .

Rovithakis, G.A., Gaganis, V.I., Perrakis, S.E. and Christodoulou, M.A., 1999. Real-time control of manufacturing cells using dynamic neural network. Automatica, 35 (1), $139-149$.

Sanghoon, L. and Mooyoung, J., 2003. Timing constraints' optimization of reserved tasks in the distributed shop-floor scheduling. International Journal of Production Research, 41 (2), 397 410.

Sugeno, M., 1999. On stability of fuzzy systems expressed by fuzzy rules with singleton consequents. IEEE Transactions on Fuzzy set and Systems, 7 (2), 201 - 224.

Tamani, K., Boukezzoula, R. and Habchi, G., 2009. Intelligent distributed and supervised flow control methodology for production systems. Engineering Applications of Artificial Intelligence, 22(7), p. $1104-1116$.

Trentesaux, D., 2009. Distributed control of production systems. Engineering Applications of Artificial Intelligence, 22(7), p. $971-978$.

Tsourveloudis, N.C., Doitsidis, L. and Ioannidis, S., 2007. Work-in-process scheduling by evolutionary tuned fuzzy controllers. International Journal of Advanced Manufacturing Technology, 34 (7-8), $748-761$.

Wiendahl, H.P. and Breithaupt, J.W., 2000. Automatic production control applying control theory. International Journal of Production Economics, 63 (1), $33-46$. 